\title{
Lessons from tracheal tube development for understanding congenital tracheal malformations
}

\author{
Heena Kumra ${ }^{1,3}$, Neha E.H. Dinesh ${ }^{1,3}$ and Dieter P. Reinhardt (10 ${ }^{1,2}$ \\ Affiliations: ${ }^{1}$ Faculty of Medicine, Dept of Anatomy and Cell Biology, McGill University, Montreal, QC, Canada. \\ ${ }^{2}$ Faculty of Dentistry, McGill University, Montreal, QC, Canada. ${ }^{3}$ Both authors contributed equally. \\ Correspondence: Dieter P. Reinhardt, Dept of Anatomy and Cell Biology, McGill University, 3640 University \\ Street, Montreal, Quebec H3A 0C7, Canada. E-mail: dieter.reinhardtamcgill.ca
}

@ERSpublications

Novel functions of the extracellular protein fibrillin-2 were discovered in tracheal tube development: fibrillin-2 has important roles in tracheal cartilage development and smooth muscle cell orientation and polarity, all required for tracheal contraction http://ow.ly/rn3Z30nId3N

Cite this article as: Kumra H, Dinesh NEH, Reinhardt DP. Lessons from tracheal tube development for understanding congenital tracheal malformations. Eur Respir J 2019; 53: 1900127 [https://doi.org/10.1183/ 13993003.00127-2019].

Fibrillins constitute a family of extracellular proteins critical for the biogenesis of elastic fibres and for the activity regulation of growth factors of the transforming growth factor (TGF)- $\beta$ superfamily. All three fibrillins are present during development of tissues and organs, including lung, aorta, bones and skin [1-3]. Typically, fibrillin-2 and -3 expression is limited to prenatal and early postnatal development in humans, whereas fibrillin-1 expression persists throughout adulthood. In mouse, the situation is simplified by the fact that fibrillin-3 is not expressed due to chromosomal rearrangement events [4]. In the developing mouse embryo, fibrillin-1 and -2 co-distribute in elastic and non-elastic tissues, with fibrillin-2 accumulating preferentially in elastic fibre-rich matrices [1]. Unlike fibrillin-1, the specific roles of fibrillin-2 (and fibrillin-3 in humans) in tissue development and homeostasis as well as in disease is still relatively little explored.

Fibrillins assemble into beaded microfibrils through homotypic or heterotypic head-to-tail and lateral self-assembly mechanisms [5-8]. Microfibrils appear to incorporate the fibrillin isoforms that are present at the time of assembly [9]. Fibrillin-2 can also persist as an inner core within postnatal microfibrils [10]. Microfibrils serve as a scaffold for the assembly of elastic fibres, which provide elasticity to various soft tissues such as lung, blood vessels and skin [11]. Mutations in the fibrillin-2 gene (FBN2) cause an autosomal dominant connective tissue disorder, congenital contractural arachnodactyly, characterised by arachnodactyly, congenital contractures, kyphoscoliosis and camptodactyly [12, 13]. A loss-of-function mutation in Fbn2 results in syndactyly in mice [14, 15]. This correlates with the radiation-induced mouse mutants "shaker with syndactylism", which is a multigene deletion that includes the Fbn2 locus $[14,16,17]$.

In the current issue of the European Respiratory Journal, YIN et al. [18] have employed forward genetic screening and analysis in mice to identify novel functions of fibrillin-2 in tracheal tubulogenesis (figure 1). In this study, loss of fibrillin-2 resulted in compromised tracheal tubulogenesis, characterised by a shortened tracheal tube as well as fewer and fragmented cartilage rings. This phenotype was caused by defective chondrocyte differentiation, whereas chondrocyte proliferation and apoptosis were unaffected. Fibrillin-2 is expressed by tracheal chondroblasts early during embryogenesis, and expression decreases over time. These findings correlate with a previous cell culture study using the chondrogenic cell line 
a)

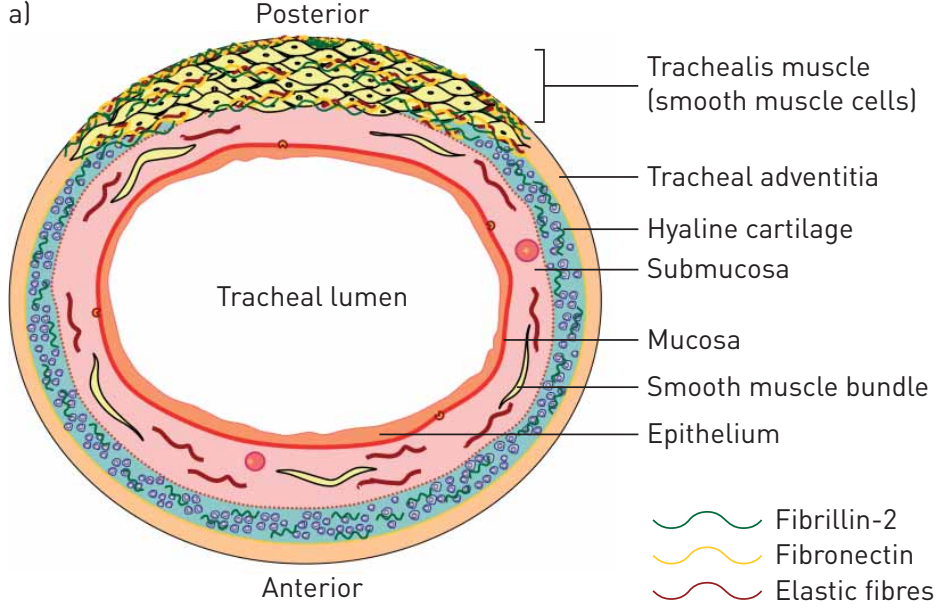

b)

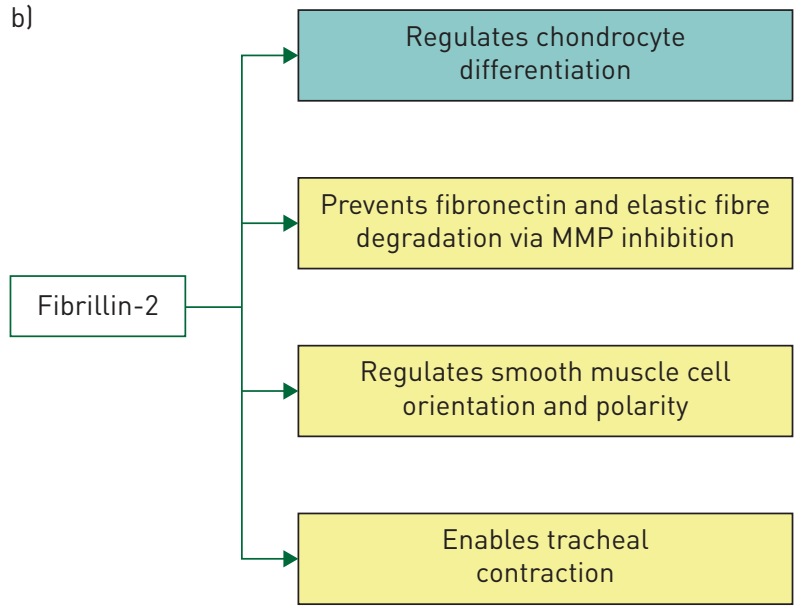

FIGURE 1 Schematic representation of a trachea in cross section, and fibrillin-2 functions in the trachea. a) The tracheal wall comprises an outermost adventitial layer, followed by a C-shaped layer of hyaline cartilage including chondroblasts/chondrocytes. Underneath the cartilage is a submucosal layer including smooth muscle bundles and elastic fibres. A thin mucosa layer separates the submucosa from an epithelium lining the tracheal lumen. The trachealis muscle bridges the gap in the cartilage, and consists of compactly packed smooth muscle cells, and extracellular matrix components. Fibrillin-2 is expressed by smooth muscle cells in the trachealis muscle, and by chondroblasts in the cartilage tissue during chondrogenic differentiation. b) Yin et al. [18] demonstrated the following functions for fibrillin-2. Fibrillin-2 aids in tracheal development by regulating chondrocyte differentiation. It also facilitates elastic fibre and fibronectin accumulation around smooth muscle cells by matrix metalloproteinase (MMP) inhibition. This leads to proper smooth muscle cell orientation and polarity. All these functional aspects of fibrillin-2 are required to support tracheal contraction. Colours of the boxes correlate with the respective colours of the tissues and proteins in a).

ATDC5, demonstrating that fibrillin-2 was highly expressed early during chondrogenesis and declined over the course of differentiation [19]. Together, these data show the importance of fibrillin-2 in early tracheal chondrogenesis. How fibrillin-2 mechanistically regulates chondrogenesis remains to be determined.

The study by YIN et al. [18] also deciphers a function of fibrillin-2 in the regulation of smooth muscle cell orientation and polarity in the trachea. The authors demonstrate that p38 mitogen-activated protein kinase (MAPK)-mediated downregulation of matrix metalloproteinases (MMPs) is instrumental for this function. More specifically, reduction of fibrillin-2 in the trachea resulted in elevated p38 MAPK phosphorylation, which in turn led to an upregulation of MMP-2 and -9, key elastin and fibronectin cleaving proteases [20]. The authors describe enhanced degradation of extracellular matrix proteins (elastin and fibronectin) around smooth muscle cells as the leading cause for the improper positioning of smooth muscle cells in the trachea, correlating well with one of the fundamental functions of the extracellular matrix being to provide environmental cues to the embedded cells [21]. The improper orientation of smooth muscle cells, and possibly the defective chondrogenesis, also led to reduced contractility of the trachea, represented by a decreased amplitude of spontaneous contractions and weakened contractile forces.

It is interesting that the role of fibrillin-2 is specific for tracheal tube development and is not observed in other organ tube development. YIN et al. [18] have shown the absence of a phenotype in the oesophagus of the Fbn2 mutant mice, which also expresses fibrillin-1. Previous studies have demonstrated the absence of a phenotype in the vasculature of $\mathrm{Fbn} 2$ null mice in the presence of fibrillin-1 [22]. Presumably, fibrillin-1 in these tissues can rescue the functions of fibrillin-2. In the vasculature, loss of fibrillin-1 in $\mathrm{Fbn1}^{--}$mice results in similar phenotypes to those of the trachea in Fbn2 mutant mice, with smooth muscle cell disorientation, upregulation of p38 MAPK and MMP levels, and elastin degradation due to a loss of contact between the cells and the matrix [18, 22, 23]. Altogether, these data illustrate that fibrillin-1 can fully compensate for the loss of fibrillin-2 in the vasculature and the oesophagus, whereas cell and tissue deficiencies can develop in the trachea, presumably due to the absence of fibrillin-1.

YIN et al. [18] also connect the findings obtained with the Fbn2 mutant mice with individuals suffering from tracheomalacia. Fibrillin-2 levels are reduced in the ventral parts of the trachea obtained from patients. It is important to note that administration of a p38 MAPK inhibitor or MMP inhibitors in the Fbn2 mutant mice partially rescued some defects in tracheal tube development. Therefore, administration of p38 MAPK inhibitors such as ralimetinib [24] or pyridinyl-imidazol compounds [25], or MMP inhibitors such as doxycycline [26], marimastat [27] or batimastat [28], may be a useful avenue to explore in the future to improve the severity of tracheomalacia in patients.

Fibrillin-containing microfibrils serve as important regulators of TGF- $\beta$ bioavailability. Elevated active TGF- $\beta$ has been correlated with disease progression in disorders caused by fibrillin-1 deficiency [29]. 
Fibrillins also adopt a crucial role in bone morphogenetic protein (BMP) signalling. The N-terminal region of fibrillin-1 serves as a docking site for the prodomains of BMP-2, -4, -7 and -10 and also imparts latency to BMP-7 [30-32]. Additionally, fibrillin-2 and BMP-7 were identified in the same genetic pathway regulating limb and muscle development, as demonstrated in mouse studies [15, 33]. Given that some BMPs have been implicated in tracheal development, exploring how BMP growth factor bioavailability and activity are affected after the loss of fibrillin-2, and how this influences tracheal tube development, might provide additional important details of the mechanism in the future.

Overall, this study illuminated a novel function of fibrillin-2 in tracheal tube development and identified mechanisms by which cartilage development and smooth muscle cell orientation influence tracheal tube formation, elongation and contraction. This basic research also provided important leads that can be potentially further explored in future clinical studies with patients suffering from tracheomalacia and congenital tracheal stenosis.

\section{Conflict of interest: None declared.}

Support statement: Funding was received from the Canadian Institutes of Health Research and the Natural Sciences and Engineering Research Council of Canada. Funding information for this article has been deposited with the Crossref Funder Registry.

\section{References}

1 Zhang $\mathrm{H}$, Apfelroth SD, Hu W, et al. Structure and expression of fibrillin-2, a novel microfibrillar component preferentially located in elastic matrices. J Cell Biol 1994; 124: 855-863.

2 Quondamatteo F, Reinhardt DP, Charbonneau NL, et al. Fibrillin-1 and fibrillin-2 in human embryonic and early fetal development. Matrix Biol 2002; 21: 637-646.

3 Sabatier L, Miosge N, Hubmacher D, et al. Fibrillin-3 expression in human development. Matrix Biol 2011; 30: 43-52.

4 Corson GM, Charbonneau NL, Keene DR, et al. Differential expression of fibrillin-3 adds to microfibril variety in human and avian, but not rodent, connective tissues. Genomics 2004; 83: 461-472.

5 Lin G, Tiedemann K, Vollbrandt T, et al. Homo- and heterotypic fibrillin-1 and -2 interactions constitute the basis for the assembly of microfibrils. J Biol Chem 2002; 277: 50795-50804.

6 Charbonneau NL, Dzamba BJ, Ono RN, et al. Fibrillins can co-assemble in fibrils, but fibrillin fibril composition displays cell-specific differences. J Biol Chem 2003; 278: 2740-2749.

7 Ashworth JL, Kelly V, Wilson R, et al. Fibrillin assembly: dimer formation mediated by amino-terminal sequences. J Cell Sci 1999; 112: 3549-3558.

8 Hubmacher D, El-Hallous EI, Nelea V, et al. Biogenesis of extracellular microfibrils: multimerization of the fibrillin-1 C terminus into bead-like structures enables self-assembly. Proc Natl Acad Sci USA 2008; 105: 6548-6553.

9 Beene LC, Wang LW, Hubmacher D, et al. Nonselective assembly of fibrillin 1 and fibrillin 2 in the rodent ocular zonule and in cultured cells: implications for Marfan syndrome. Invest Ophthalmol Vis Sci 2013; 54: 8337-8344.

10 Charbonneau NL, Jordan CD, Keene DR, et al. Microfibril structure masks fibrillin-2 in postnatal tissues. J Biol Chem 2010; 285: 20242-20251.

11 Hubmacher D, Reinhardt DP. Microfibrils and fibrillin. In: Mecham RP, ed. The Extracellular Matrix: an Overview (Biology of Extracellular Matrix). New York, Springer, 2011; pp. 233-265.

12 Gupta PA, Putnam EA, Carmical SG, et al. Ten novel FBN2 mutations in congenital contractural arachnodactyly: delineation of the molecular pathogenesis and clinical phenotype. Hum Mutat 2002; 19: 39-48.

13 Putnam EA, Zhang H, Ramirez F, et al. Fibrillin-2 (FBN2) mutations result in the Marfan-like disorder, congenital contractural arachnodactyly. Nat Genet 1995; 11: 456-458.

14 Chaudhry SS, Gazzard J, Baldock C, et al. Mutation of the gene encoding fibrillin-2 results in syndactyly in mice. Hum Mol Genet 2001; 10: 835-843.

15 Arteaga-Solis E, Gayraud B, Lee SY, et al. Regulation of limb patterning by extracellular microfibrils. J Cell Biol 2001; 154: 275-281.

16 Hertwig P. Neue Mutationen und Koppelungsgruppen bei der Hausmaus [New mutations and coupling groups in the house mouse]. Z Indukt Abstamm Vererbungsl 1942; 80: 220-246.

17 Johnson KR, Cook SA, Zheng QY. The original shaker-with-syndactylism mutation (sy) is a contiguous gene deletion syndrome. Mamm Genome 1998; 9: 889-892.

18 Yin W, Kim H-T, Wang SP, et al. Fibrillin-2 is a key mediator of smooth muscle extracellular matrix homeostasis during mouse tracheal tubulogenesis. Eur Respir J 2019; 53: 1800840.

19 Yanagino T, Yuasa K, Nagahama M, et al. Transcriptional regulation of fibrillin-2 gene by E2F family members in chondrocyte differentiation. J Cell Biochem 2009; 106: 580-588.

20 Hsu CC, Lai SC. Matrix metalloproteinase-2, -9 and -13 are involved in fibronectin degradation of rat lung granulomatous fibrosis caused by Angiostrongylus cantonensis. Int J Exp Pathol 2007; 88: 437-443.

21 Frantz C, Stewart KM, Weaver VM. The extracellular matrix at a glance. J Cell Sci 2010; 123: 4195-4200.

22 Carta L, Pereira L, Arteaga-Solis E, et al. Fibrillins 1 and 2 perform partially overlapping functions during aortic development. J Biol Chem 2006; 281: 8016-8023.

23 Carta L, Smaldone S, Zilberberg L, et al. p38 MAPK is an early determinant of promiscuous Smad2/3 signaling in the aortas of fibrillin-1 (Fbn1)-null mice. J Biol Chem 2009; 284: 5630-5636.

24 Patnaik A, Haluska P, Tolcher AW, et al. A first-in-human phase I study of the oral p38 MAPK inhibitor, ralimetinib (LY2228820 dimesylate), in patients with advanced cancer. Clin Cancer Res 2016; 22: 1095-1102.

25 Tong L, Pav S, White DM, et al. A highly specific inhibitor of human p38 MAP kinase binds in the ATP pocket. Nat Struct Biol 1997; 4: 311-316. 
26 Lindeman JH, Abdul-Hussien $\mathrm{H}$, van Bockel JH, et al. Clinical trial of doxycycline for matrix metalloproteinase-9 inhibition in patients with an abdominal aneurysm: doxycycline selectively depletes aortic wall neutrophils and cytotoxic T cells. Circulation 2009; 119: 2209-2216.

27 Thompson RW, Baxter BT. MMP inhibition in abdominal aortic aneurysms. Rationale for a prospective randomized clinical trial. Ann N Y Acad Sci 1999; 878: 159-178.

28 Treharne GD, Boyle JR, Goodall S, et al. Marimastat inhibits elastin degradation and matrix metalloproteinase 2 activity in a model of aneurysm disease. Br J Surg 1999; 86: 1053-1058.

29 Neptune ER, Frischmeyer PA, Arking DE, et al. Dysregulation of TGF- $\beta$ activation contributes to pathogenesis in Marfan syndrome. Nat Genet 2003; 33: 407-411.

30 Sengle G, Charbonneau NL, Ono RN, et al. Targeting of bone morphogenetic protein growth factor complexes to fibrillin. J Biol Chem 2008; 283: 13874-13888.

31 Sengle G, Ono RN, Sasaki T, et al. Prodomains of transforming growth factor $\beta$ (TGF $\beta$ ) superfamily members specify different functions: extracellular matrix interactions and growth factor bioavailability. J Biol Chem 2011; 286: 5087-5099.

32 Wohl AP, Troilo H, Collins RF, et al. Extracellular regulation of bone morphogenetic protein activity by the microfibril component fibrillin-1. J Biol Chem 2016; 291: 12732-12746.

33 Sengle G, Carlberg V, Tufa SF, et al. Abnormal activation of BMP signaling causes myopathy in Fbn2 null mice. PLoS Genet 2015; 11: e1005340. 